\title{
Blood pressure reduction combining baroreflex restoration for stroke prevention in hypertension in rats
}

\author{
Shu-Wei Song ${ }^{1,2+}$, Ai-Jun Liu' ${ }^{1+}$, Chong Bai ${ }^{3}$, Bei-Lin Su', Xiu-Juan Ma', Fu-Ming Shen', Jun-Li Duan ${ }^{4 *}$ and \\ Ding-Feng Su ${ }^{1 *}$
}

\author{
Department of Pharmacology, School of Pharmacy, Second Military Medical University, Shanghai, China \\ 2 Department of Nephrology, Changzheng Hospital, Kidney Center of PLA, Second Military Medical University, Shanghai, China \\ ${ }^{3}$ Department of Respiratory Diseases, Second Military Medical University, Shanghai, China \\ ${ }^{4}$ Department of Gerontology, Xinhua Hospital, Shanghai Jiaotong University, Shanghai, China
}

Edited by:

Issy Laher, University of British

Columbia, Canada

Reviewed by:

Marilyn J. Cipolla, University of Vermont, USA

George C. Wellman, University of

Vermont, USA

\section{*Correspondence:}

Ding-Feng Su, Department of

Pharmacology, School of Pharmacy,

Second Military Medical University, 325

Guo He Road, Shanghai 200433, China.

e-mail:dfsu2008@gmail.com,

Jun-Li Duan, Department of

Gerontology, Xinhua Hospital,

Shanghai Jiaotong University, Shanghai

200003, China

e-mail: Junlishanghai2005@yahoo.

com.cn

${ }^{+}$Shu-Wei Song and Ai-Jun Liu

contributed equally to this work.
Blood pressure reduction is an important and effective strategy in stroke prevention in hypertensives. Recently, we found that baroreflex restoration was also crucial in stroke prevention. The present work was designed to test the hypothesis that a combination of blood pressure reduction and baroreflex restoration may be a new strategy for stroke prevention. In Experiment 1, the effects of ketanserin $(0.3,1,3,10 \mathrm{mg} / \mathrm{kg})$, amlodipine $(0.3,1,2,3 \mathrm{mg} / \mathrm{kg})$ and their combination $(1+0.3$, $1+1,1+2,1+3 \mathrm{mg} / \mathrm{kg}$ ) on blood pressure and baroreflex sensitivity (BRS) of stroke-prone spontaneously hypertensive rats (SHR-SP) were determined under conscious state. It was found that both amlodipine and ketanserin decreased blood pressure dose-dependently. Ketanserin enfanced BRS from a very small dose but amlodipine enfanced BRS only at largest dose used. At the dose of $1+2 \mathrm{mg} / \mathrm{kg}$ (ketanserin + amlodipine), the combination possessed the largest synergism on blood pressure reduction. In Experiments 2 and 3, SHR-SP and two-kidney, two-clip $(2 \mathrm{~K} 2 \mathrm{C})$ renovascular hypertensive rats received life-long treatments with ketanserin $(1 \mathrm{mg} / \mathrm{kg})$ and amlodipine $(2 \mathrm{mg} / \mathrm{kg})$ or their combination $(0.5+1,1+2,2+4 \mathrm{mg} / \mathrm{kg})$. The survival time was recorded and the brain lesion was examined. It was found that all kinds of treatments prolonged the survival time of SHR-SP and $2 \mathrm{~K} 2 \mathrm{C}$ rats. The combination possessed a significantly better effect on stroke prevention than mono-therapies. In conclusion, combination of blood pressure reduction and baroreflex restoration may be a new strategy for the prevention of stroke in hypertension.

Keywords: stroke, stroke-prone spontaneously hypertensive rats, two-kidney, two-clip renovascular hypertensive rats, hypertension, arterial baroreflex, prevention

\section{INTRODUCTION}

According to recent reports published by the World Health Organization, about 15 million people per year fall victim to stroke, one-third of whom die and another third are left permanently disabled. Therefore, prevention is the only possible way to curb the stroke pandemic (Franco et al., 2004a,b; Papadopoulos and Papademetriou, 2008). Hypertension is the most important modifiable risk for stroke. It is reported that over $70 \%$ of stroke can be attributed to hypertension (Papadopoulos and Papademetriou, 2008). Blood pressure control is an important way to reduce the morbidity of stroke.

However, it is well accepted that blood pressure level is not the unique determinant for stroke in hypertension. Recently, we found that arterial baroreflex function, expressed as baroreflex sensitivity (BRS), is another important determinant for stroke (Liu et al., 2007). Stroke-prone spontaneously hypertensive rats (SHR-SP) were divided into two groups according to their BRS at age of 6 months. It was found that stroke was significantly delayed in rats with high BRS compared with those with low BRS (Liu et al., 2007).

Based on these findings, we speculated that a combination of blood pressure reduction and BRS restoration might be much beneficial for preventing stroke in hypertension. The present work was therefore designed to test this hypothesis. Amlodipine was used for blood pressure reduction and a small dose of ketanserin for BRS restoration. In this work, we used not only SHR-SP, but also a new spontaneous stroke model, two-kidney, two-clip (2K2C) renovascular hypertensive rats.

\section{MATERIALS AND METHODS ANIMALS}

Sprague-Dawley (SD) rats were provided by Sino-British SIPPR/ BK Lab Animal Ltd (Shanghai, China) and SHR-SP by the Animal Center of our university. They were housed with controlled temperature $\left(23-25^{\circ} \mathrm{C}\right)$ and lighting (8:00-20:00 light, 20:00-8:00 dark) and with free access to standard food and drinking water. All the animals used in this work received humane care in compliance with institutional guidelines for health and care of experimental animals.

\section{BLOOD PRESSURE MEASUREMENT}

Systolic blood pressure (SBP), diastolic blood pressure (DBP) and heart period were continuously recorded using previously described technique with some modifications (Su et al., 1986). Briefly, rats were anesthetized with a combination of ketamine and diazepam. A polyethylene catheter was inserted into the lower abdominal aorta via the left femoral artery for blood pressure measurement and another catheter was inserted into left femoral vein for phenylephrine administration. A third catheter was placed into the stomach via a mid-abdominal incision for drug administration. The catheters were exteriorized through the interscapular skin. After 2-day recovery period, the animals were placed for blood pressure recording in individual cylindrical cages containing food and water. The aortic catheter was connected to a blood pressure transducer via a rotating swivel that allowed the animals to move freely in the 
cage. After about 4-h habituation, the blood pressure signal was digitized by a microcomputer. SBP, DBP and heart period values were determined on line. The mean values of these parameters during a period of given time were calculated.

\section{DETERMINATION OF BRS}

In the above-mentioned blood pressure recording condition, BRS was measured in the conscious rat (Zhang et al., 2008). A bolus injection of phenylephrine was used to induce a blood pressure elevation. The dose of phenylephrine $(5-10 \mu \mathrm{g} / \mathrm{kg})$ was adjusted to raise SBP about $30 \mathrm{mmHg}$. There exists a delay (about $1 \mathrm{~s}$ ) between the elevation of blood pressure (stimulus) and the prolongation of heart period (response) for arterial baroreflex. In rats, the heart rate is about 5 or $6 \mathrm{~s}$. So, heart period was plotted against SBP for linear regression analysis for 2-8 shifts (calculated by computer); the slope with the largest correlation coefficient $(r)$ of heart period/SBP was expressed as BRS ( $\mathrm{ms} / \mathrm{mm} \mathrm{Hg}$ ). The mean of two measurements with proper dose was taken as the final result.

\section{PREPARATION OF 2K2C RENOVASCULAR HYPERTENSIVE RATS}

Male SD rats weighing 190-210 g were anesthetized with a combination of ketamine $(40 \mathrm{mg} / \mathrm{kg})$ and diazepam $(6 \mathrm{mg} / \mathrm{kg})$. A median longitudinal incision on abdominal skin was performed, and then two silver clips (0.3-mm internal gap) were placed on the roots of both right and left renal arteries (Zeng et al., 1998). All animals were fed standard rat chow and tap water ad libitum. One month after operation, SBP was measured by tail-cuff plethysmography.

\section{STROKE SYMPTOM OBSERVATION}

To detect the stroke symptoms, the movement of limbs, respiration, diet, fur, and consciousness of all SHR-SP and 2K2C hypertensive rats were observed twice daily (at 8:00 and 18:00).

\section{MORPHOLOGICAL EXAMINATION}

In experiment 2 and 3, when the rats died, the brain was removed, and the signs of hemorrhage and ischemia were examined, and then photographed. Brains without these distinct signs were immersed in the solution of $4 \%$ paraformaldehyde in $0.1 \mathrm{~mol} / \mathrm{L}$ phosphate buffer ( $\mathrm{pH}$ 7.4), and then the brain was dissected and fixed in this solution for $24 \mathrm{~h}$. The specimens were then washed, dehydrated in a graded ethanol series, and embedded in paraffin. Sections cut transversely at 5 - $\mu \mathrm{m}$ thickness separated by $100 \mu \mathrm{m}$ from the anterior to the posterior extremity were stained with hematoxylin and eosin for light microscopic study (Xie et al., 2005).

\section{PROBABILITY SUM TEST}

To determine whether the combination was synergistic, we used the probability sum test ( $q$ test) (Xie et al., 2005). This test came from classic probability analysis and was used for evaluating the synergism of the combination of two drugs. In the present work, we used the following criteria. Compared with the mean values of baseline (before drug administration), rats with a decrease of the mean value over $4 \mathrm{~h} \geq 20 \mathrm{mmHg}$ in SBP were defined as responders. The formula is as follows: $q=P_{\mathrm{A}+\mathrm{B}} /\left(P_{\mathrm{A}}+P_{\mathrm{B}}-P_{\mathrm{A}} \times P_{\mathrm{B}}\right)$. Here, $\mathrm{A}$ and $\mathrm{B}$ indicate drug $A$ and drug $B ; P$ (probability) is the percentage of responders in each group. $P_{\mathrm{A}+\mathrm{B}}$ is real percentage of responder and $\left(P_{\mathrm{A}}+P_{\mathrm{B}}-P_{\mathrm{A}} \times P_{\mathrm{B}}\right)$ is expected response rate. $\left(P_{\mathrm{A}}+P_{\mathrm{B}}\right)$ indicates the sum of the probabilities when drug $\mathrm{A}$ and drug $\mathrm{B}$ is used alone. $\left(P_{\mathrm{A}} \times P_{\mathrm{B}}\right)$ is the probability of rats responding to both drugs when they were used alone. When $q<0.85$ the combination is antagonistic; when $q>1.15$ it is synergistic. It is additive when $q$ is between 0.85 and 1.15 .

\section{PROTOCOL}

\section{Experiment 1}

Effects of amlodipine, ketanserin and their combination on blood pressure and BRS in SHR-SP. In this experiment, 108 female SHR-SP aged 8 months were used. They were randomly divided into 12 groups ( $n=9$ in each group). After approximately 4-h habituation (from 8:00 to $12: 00$ ), blood pressure was recorded during a period of $30 \mathrm{~min}$ (from 12:00 to 12:30) and BRS was measured using the previously mentioned method. These values were taken as basal control values. After the determination of baseline values, each group of rats received intragastrically ketanserin $(0.3,1,3$ and $10 \mathrm{mg} / \mathrm{kg})$, amlodipine $(0.3,1,2,3 \mathrm{mg} / \mathrm{kg})$, or their combination $(1+0.3,1+1,1+2,1+3 \mathrm{mg} / \mathrm{kg})$ respectively. Approximately $60 \mathrm{~min}$ after drug administration, blood pressure was recorded for another $30 \mathrm{~min}$ and BRS was determined again.

\section{Experiment 2}

Effects of amlodipine, ketanserin and their combination on stroke prevention in SHR-SP. Ninety male SHR-SP, aged 5 months, were randomly divided into six groups ( $n=15$ in each group). Drugs were mixed in the chow. The content of drugs in the rat chow was calculated according to consumption. The daily-ingested doses were as follows (shown in Table 1). The stroke occurrences, stroke subtypes and death time were recorded.

\section{Experiment 3}

Effects of amlodipine, ketanserin and their combination on stroke prevention in $2 \mathrm{~K} 2 \mathrm{C}$ rats. Total 120 male SD rats were randomly divided into six groups ( $n=20$ in each group). One month after $2 \mathrm{~K} 2 \mathrm{C}$ operation, blood pressures were measured by tail-cuff plethysmography. Then these rats received treatments. The treatments were exactly the same as above-mentioned for SHR-SP (Table 1). Dead rats without stroke, but with obvious abnormity on other organs such as heart, lung, or kidney, were excluded from the protocol. The stroke occurrences, stroke subtypes and death time were recorded.

\section{STATISTICAL ANALYSIS}

Investigators were blind to the procedures during blood pressure and heart period recording, BRS determination, weighing and morphological examination. Data are expressed as the mean \pm SE. The comparisons between pre- and post-drug were made by paired $t$-tests.

\section{Table 1 |The doses of ketanserin and amlodipine in six groups.}

\begin{tabular}{lll}
\hline Group & \multicolumn{2}{c}{ Dose $(\mathbf{m g} / \mathbf{k g} / \mathbf{d})$} \\
\cline { 2 - 3 } & Ketanserin & Amlodipine \\
\hline 1 & 0 & 0 \\
2 & 1 & 0 \\
3 & 0 & 2 \\
4 & 0.5 & 1 \\
5 & 1 & 2 \\
6 & 2 & 4
\end{tabular}


In experiments 2 and 3, Kaplan-Meier analysis was used to estimate survival probabilities. Log-Rank testing was used to evaluate equality of survival curves. $P<0.05$ was considered statistically significant.

\section{RESULTS}

\section{EFFECTS OF AMLODIPINE, KETANSERIN AND THEIR COMBINATION ON} BLOOD PRESSURE AND BRS IN SHR-SP

The effects of a single dose of ketanserin and amlodipine, either alone or in combination, on blood pressure, heart period, and BRS are shown in Figure 1. It was found that from $0.3 \mathrm{mg} / \mathrm{kg}$ to
$3.0 \mathrm{mg} / \mathrm{kg}$, amlodipine significantly decreased SBP and DBP in a dose-dependent manner. The maximal decrease $(-22 \mathrm{mmHg})$ in SBP was found with $3 \mathrm{mg} / \mathrm{kg}$. A significant decrease in heart period (increase in heart rate) existed with the doses from $1.0 \mathrm{mg} / \mathrm{kg}$. A similar result was seen on BRS. Amlodipine enhanced BRS at the doses 1, 2, and $3 \mathrm{mg} / \mathrm{kg}$. No effects on blood pressure were found when ketanserin was given at $0.3 \mathrm{mg} / \mathrm{kg}$. From $1.0 \mathrm{mg} / \mathrm{kg}$, ketanserin significantly decreased both SBP and DBP. It seems that the decrease in DBP was more profound than the decrease in SBP. Ketanserin did not affect heart period. The most important phenomenon is the effect
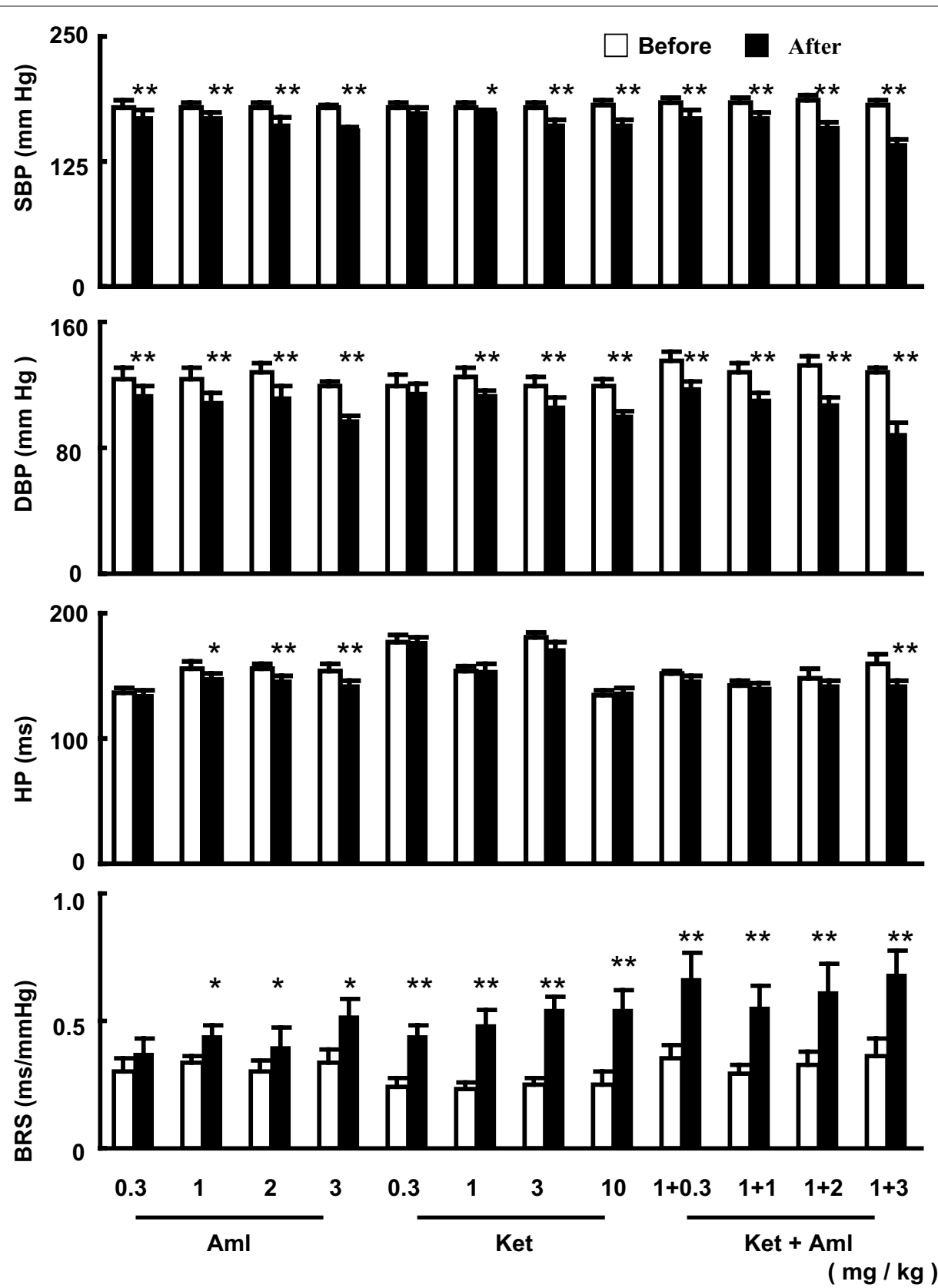

FIGURE 1 |The effects of a single dose of amlodipine (Aml) and ketanserin (Ket) or combination on systolic blood pressure (SBP), diastolic blood pressure (DBP), heart period (HP) and baroreflex sensitivity (BRS) in conscious SHR-SP. The values are expressed as mean \pm SE. ${ }^{*} P<0.05$, ${ }^{*} P<0.01$ after drug vS. before drug. $n=9$ in each group. 
of ketanserin on BRS. Even at very-low dose, ketanserin increased significantly BRS in SHR-SP. The effect of ketanserin on BRS was neither dose-dependent nor blood pressure dependent (Figure 1). In combination studies, the dose of ketanserin was fixed at $1.0 \mathrm{mg} / \mathrm{kg}$. Ketanserin was combined with four different doses of amlodipine. It was found that the effects of the combinations on blood pressure were enhanced with the increase of the doses of amlodipine. However, this phenomenon was not found on BRS. The combination with the smallest dose of amlodipine had already a great effect.

The synergism of the combination was determined by $q$ test. The $q$-test results are shown in Table 2. It is clear that there was a significant synergism on blood pressure reduction for the combination of ketanserin and amlodipine. The best ratio of this combination was $1: 2$. When $1 \mathrm{mg} / \mathrm{kg}$ ketanserin combined with $2 \mathrm{mg} / \mathrm{kg}$ amlodipine, we arrived at $q$ values of 2.4 for SBP and 2.1 for DBP for the combination of ketanserin and amlodipine. But there was no synergistic interaction of ketanserin and amlodipine on BRS. The $q$ values were all lower than 1.15 (1.04-1.09) and exhibited an additive effect.

Table 2 |The probability sum test in SHR-SP treated with ketanserin (Ket) and amlodipine (Aml) Alone or in combination.

\begin{tabular}{|c|c|c|c|}
\hline Parameter & Dose $(\mathrm{mg} / \mathbf{k g})$ & $P$ value (\%) & $q$ value \\
\hline \multicolumn{4}{|l|}{ SBP } \\
\hline Ket & 1 & 0 & \\
\hline Aml & 0.3 & 0 & \\
\hline Aml & 1 & 0 & \\
\hline Aml & 2 & 33 & \\
\hline Aml & 3 & 50 & \\
\hline Ket + Aml & $1+0.3$ & 29 & \\
\hline $\mathrm{Ket}+\mathrm{Aml}$ & $1+1$ & 29 & \\
\hline $\mathrm{Ket}+\mathrm{Aml}$ & $1+2$ & 80 & 2.4 \\
\hline Ket + Aml & $1+3$ & 89 & 1.8 \\
\hline \multicolumn{4}{|l|}{ DBP } \\
\hline Ket & 1 & 10 & \\
\hline Aml & 0.3 & 0 & \\
\hline Aml & 1 & 43 & \\
\hline Aml & 2 & 33 & \\
\hline Aml & 3 & 50 & \\
\hline $\mathrm{Ket}+\mathrm{Aml}$ & $1+0.3$ & 29 & \\
\hline $\mathrm{Ket}+\mathrm{Aml}$ & $1+1$ & 57 & 1.17 \\
\hline $\mathrm{Ket}+\mathrm{Aml}$ & $1+2$ & 70 & 2.1 \\
\hline $\mathrm{Ket}+\mathrm{Aml}$ & $1+3$ & 100 & 1.8 \\
\hline \multicolumn{4}{|l|}{ BRS } \\
\hline Ket & 1 & 60 & \\
\hline Aml & 0.3 & 14 & \\
\hline Aml & 1 & 14 & \\
\hline Aml & 2 & 11 & \\
\hline Aml & 3 & 50 & \\
\hline $\mathrm{Ket}+\mathrm{Aml}$ & $1+0.3$ & 71 & 1.08 \\
\hline $\mathrm{Ket}+\mathrm{Aml}$ & $1+1$ & 71 & 1.08 \\
\hline $\mathrm{Ket}+\mathrm{Aml}$ & $1+2$ & 70 & 1.09 \\
\hline Ket + Aml & $1+3$ & 83 & 1.04 \\
\hline
\end{tabular}

$P$ indicated the percentage of animals possessing an effective decrease in BP $(20 \mathrm{~mm} \mathrm{Hg})$, and an effective increase in BRS $(0.2 \mathrm{~ms} / \mathrm{mm} \mathrm{Hg})$ produced by ketanserin, amlodipine alone or in combination. $n=9$ in each group.

\section{EFFECTS OF AMLODIPINE, KETANSERIN AND THEIR COMBINATION ON STROKE PREVENTION IN SHR-SP}

Among the 90 rats studied, 88 died from stroke were confirmed by displaying neurological symptoms of stroke and/or brain pathological examination. About one-third animals died of hemorrhage and two-thirds of infarction. The subtypes of stroke are shown in Table 3. This proportion of hemorrhage and ischemia was not changed by the treatments. The survival time expressed by the Kaplan-Meier survival curve is shown in Figure 2. The average survival time in control SHR-SP was $332 \pm 66$ days. A significant difference was detected between the control and the five drug treatment groups (Log-rank testing $\chi^{2}=50.2, P<0.0001$ ). From Groups 2 to 6 (treated rats), the survival time was as follows: $398 \pm 85$ days; $404 \pm 80$ days; $424 \pm 90$ days; $470 \pm 94$ days; and $508 \pm 93$ days. Compared with control group, the survival time was 1.20 -fold greater in Group $2(P<0.05), 1.22$-fold in Group $3(P<0.05), 1.28$-fold in Group $4(P<0.01), 1.42$-fold in Group $5(P<0.001)$, and 1.53-fold in Group $6(P<0.001)$. When the control rats all died of stroke, the mortality rate in different treatment groups was as follows: $53 \%$ in Group 2; $33 \%$ in Group 3; $27 \%$ in Group 4; $20 \%$ in Group 5; and 13\% in Group 6. These data showed that the treatment can prevent the occurrence of stroke, especially the combination of amlodipine and ketanserin.

\section{EFFECTS OF AMLODIPINE, KETANSERIN AND THEIR COMBINATION ON STROKE PREVENTION IN 2K2C RENOVASCULAR HYPERTENSIVE RATS}

One month after $2 \mathrm{~K} 2 \mathrm{C}$ operation, blood pressures were measured by tail-cuff plethysmography. All $2 \mathrm{~K} 2 \mathrm{C}$ rats exhibited a SBP $>150 \mathrm{mmHg}$. Among the 120 rats studied, 81 animals died from stroke. Other 39 animals died without stroke were excluded from the protocol. The subtypes of stroke are also shown in Table 3. This proportion of hemorrhage and ischemia was not changed by the treatments. The survival time expressed by the Kaplan-Meier survival curve is shown in Figure 3.

The average survival time in control rats was $194 \pm 75$ days. A significant difference was detected between the control and the five drug treatment groups (Log-rank testing $\chi^{2}=31.2, P<0.0001$ ). From Groups 2 to 6 (treated rats), the survival time was as follows: $277 \pm 123$ days; $310 \pm 121$ days; $354 \pm 142$ days; $364 \pm 148$ days; and $381 \pm 163$ days. Compared with control group, the survival time was 1.43-fold greater in Group $2(P<0.05), 1.60$-fold in Group 3 $(P<0.01), 1.82$-fold in Group $4(P<0.0001), 1.88$-fold in Group $5(P<0.0001)$, and 1.96 -fold in Group $6(P<0.0001)$. When the control rats all died of stroke, the mortality rate in different treatment groups was as follows: 67\% in Group 2; 64\% in Group 3; $43 \%$ in Group 4; 36\% in Group 5; and 38\% in Group 6. These data also showed that the treatment can significantly prevent the occurrence of stroke, especially the combination of amlodipine and ketanserin.

\section{DISCUSSION}

It is estimated that over $70 \%$ of stroke can be attributed to hypertension. Blood pressure level is an important determinant for stroke. The risk of stroke is increased by about $25 \%$ with each $10 \mathrm{mmHg}$ increase in SBP (Wolf-Maier et al., 2003; Fang et al., 2006; Luepker et al., 2006). Accordingly, expected benefits of blood pressure lowering 
Table 3 | Occurrence of brain lesions in different groups of SHR-SP and 2K2C rats.

\begin{tabular}{|c|c|c|c|c|c|c|c|c|c|c|}
\hline Group & \multicolumn{5}{|c|}{ SHR-SP } & \multicolumn{5}{|c|}{$2 \mathrm{~K} 2 \mathrm{C}$} \\
\hline 2 & 15 & 5 & 6 & 4 & 0 & 20 & 3 & 7 & 5 & 5 \\
\hline 3 & 15 & 4 & 9 & 2 & 0 & 20 & 3 & 8 & 3 & 6 \\
\hline 4 & 15 & 4 & 8 & 3 & 0 & 20 & 2 & 7 & 5 & 6 \\
\hline 6 & 15 & 4 & 6 & 4 & 1 & 20 & 3 & 6 & 4 & 7 \\
\hline
\end{tabular}

Hem, hemorrhage; Isc, ischemia; Mix, mixture of Hem and Isc.

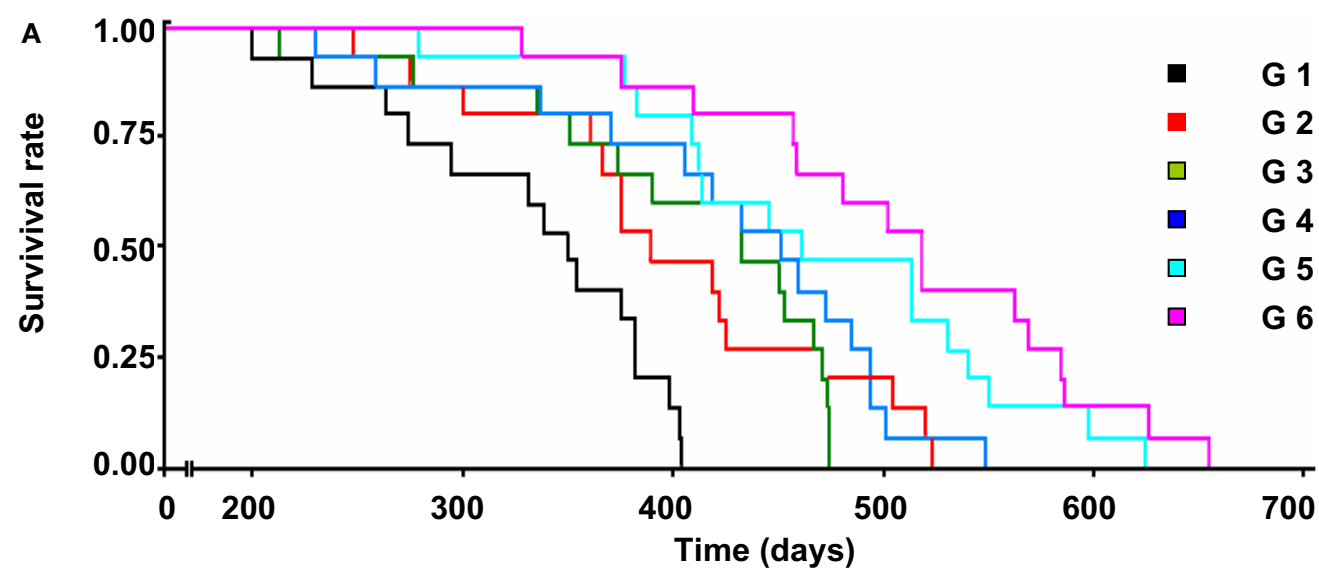

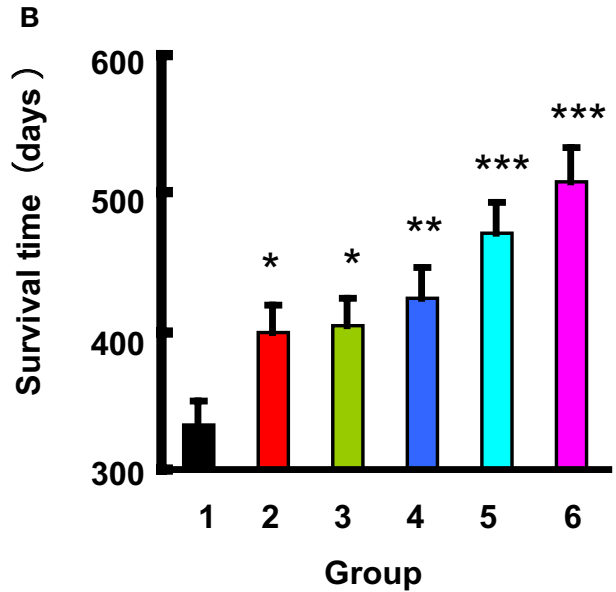

FIGURE 2 |The effects of life-long treatment of ketanserin (Ket) and amlodipine (Aml) on the stroke death in SHR-SP. (A)The survival rate in these six subgroups (G, group), doses referred to Table 1, is expressed by Kaplan-Meier
C

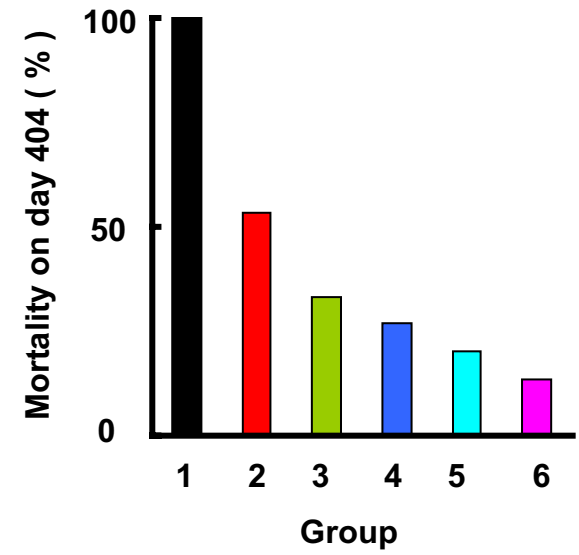

survival curves. (B) The survival time expressed as mean \pm SE. ${ }^{*} P<0.05$, ${ }^{*} P<0.01,{ }^{*} * P<0.001$ for treatment group $v s$ control group. $n=14-15$ in each group. (C) The mortality rate on day 404 , when the control rats all died. for stroke risk reduction are broadly consistent across a range of different population subgroups (Lawes et al., 2004; Papadopoulos and Papademetriou, 2008). In the present work, it was found that a reduction of blood pressure with amlodipine $(2 \mathrm{mg} / \mathrm{kg} /$ day $)$ could significantly delay the occurrence of stroke and prolonged survival time in both SHR-SP (+72 days) and $2 \mathrm{~K} 2 \mathrm{C}$ hypertensive rats (+125 days).
Many researchers have confirmed that baroreflex dysfunction was associated to the prognosis of many diseases (La Rovere et al., 1988, 1998; Robinson et al., 2003). La Rovere and colleagues assessed BRS 4 weeks after the first attack of acute myocardial infarction. During a follow-up period of 2 years, they found that the mortality rate is $40 \%$ in patients with a BRS $<3.0 \mathrm{~ms} / \mathrm{mmHg}$ while $3 \%$ in patients with a BRS $>3.0 \mathrm{~ms} / \mathrm{mmHg}$ (La Rovere et al., 

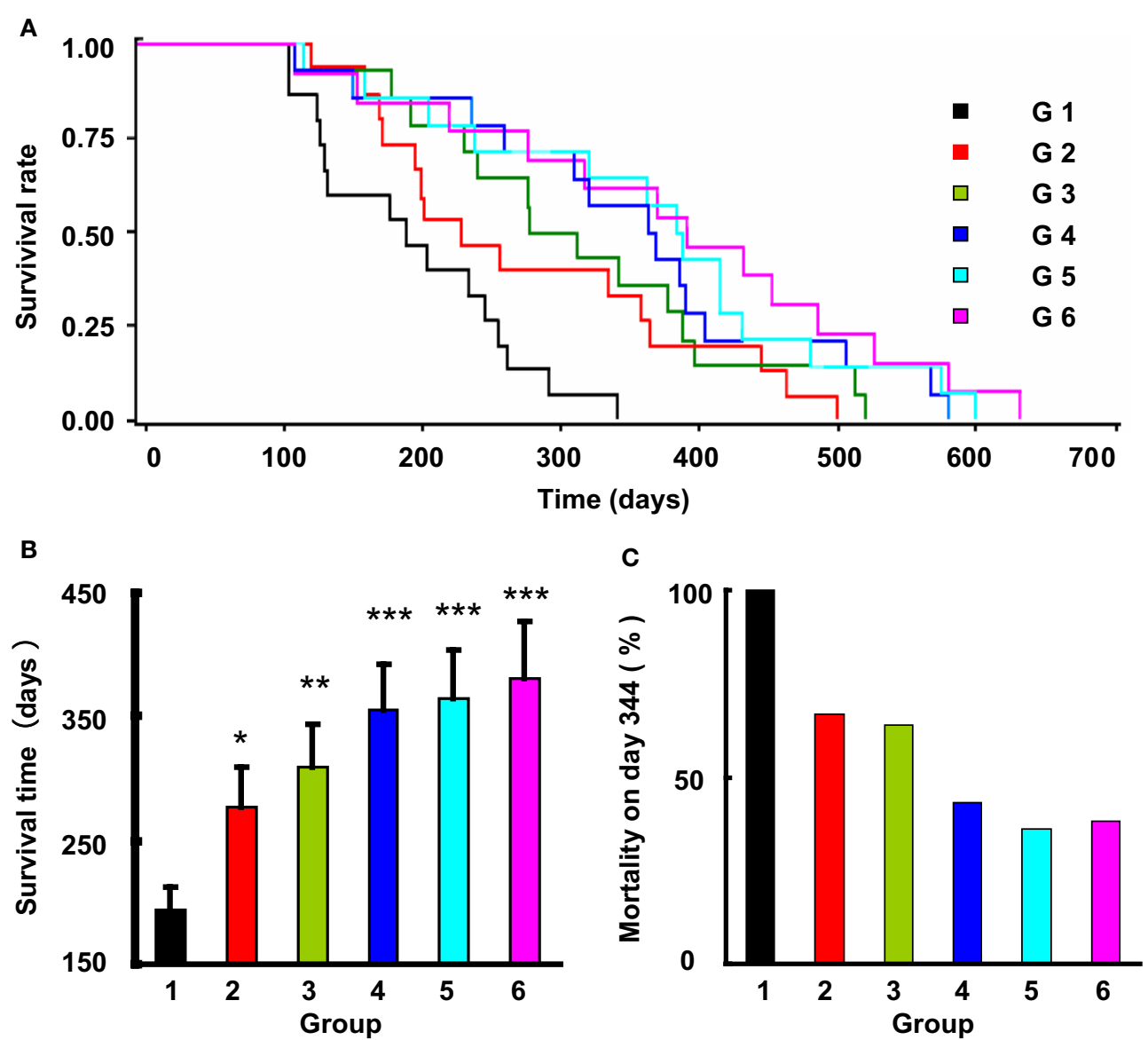

FIGURE 3 |The effects of life-long treatment of ketanserin and amlodipine on the stroke death in $2 \mathrm{~K} 2 \mathrm{C}$ rats. (A) The survival rate in these six subgroups (G, group), doses referred to Table 1, is expressed by Kaplan-Meier survival

1998). Robinson et al. (2003) reported that post-stroke patients with impaired BRS values had a significantly poorer prognosis than patients without impaired BRS. Recently, a series of studies concerning the baroreflex dysfunction and cardiovascular diseases in rats was performed in our department. It was found that baroreflex dysfunction was related to: the severity of atherosclerosis induced by high-cholesterol diet and the severity of acute cerebral ischemia induced by middle cerebral artery occlusion, the survival times in aconitine-induced ventricular arrhythmia, in endotoxin-induced shock and in SHR-SP (Cai et al., 2005; Liu et al., 2007, 2008).

Ketanserin is an antihypertensive drug with affinity for both $5-\mathrm{HT}_{2 \mathrm{~A}}$ and $\alpha_{1}$ receptors. Our previous studies demonstrated that ketanserin could effectively enhance BRS in SHR and SHR-SP (Fu et al., 2004). This effect is not blood pressure dependent as a small dose of ketanserin could enhance BRS without BP modification. In addition, this effect is mediated by central $5-\mathrm{HT}_{2 \mathrm{~A}}$ receptor $(\mathrm{Fu}$ et al., 2004, 2006; Shen et al., 2007). Previously, we demonstrated that a small dose of ketanserin which did not decrease blood pressure but enhanced BRS could effectively prolong the survival time in SHR-SP (Liu et al., 2007).
Based on above-mentioned data, the main working hypothesis of the present study is to propose that a combination of blood pressure reduction and baroreflex restoration may be as a new strategy for stroke prevention in hypertension. To test this hypothesis, some considerations are listed as follows:

(1) Drug selection. The antihypertensive effect of amlodipine is well recognized. In addition, it is believed as one of the best drugs for stroke prevention in hypertensive patients (Papadopoulos and Papademetriou, 2008). Since 20 years, we have studied many drugs for enhancing BRS. It was found that ketanserin is the most effective agent for baroreflex restoration. Therefore, amlodipine and ketanserin were selected for blood pressure reduction and baroreflex restoration respectively in this work.

(2) Dose determination. The daily dose is $5-10 \mathrm{mg}$ for amlodipine and $40 \mathrm{mg}$ for ketanserin clinically. We hope in clinic to use an ordinary dose of amlodipine and a small dose of ketanserin. Clinically, the daily dose for ketanserin is $4-8$ times greater than amlodipine. In the present work, ketanserin: amlodipine is $1: 2$. This means if we use an ordinary 
dose for amlodipine in clinic, that the dose of ketanserin is only $1 / 8$ to $1 / 16$ of the ordinary dose. It is a very small dose and this will be beneficial to patients requiring life-long treatment as the possible side effects will be minimized by such a small dose.

(3) Animal models. SHR-SP is the best genetic animal model for studying the mechanism of the genesis of stroke and for testing the effectiveness of drugs in stroke prevention (Hamaguchi et al., 2008; Wang et al., 2008; Daneshtalab and Smeda, 2010; Henning et al., 2010). It is widely used and was certainly included in the present work. In addition, we used also another animal model, $2 \mathrm{~K} 2 \mathrm{C}$ renovascular hypertensive rats. This non-genetic model was firstly established by Zeng et al. (1998). The incidence of hypertension is $100 \%$ and the incidence of stroke is higher than $60 \%$ within 10 months after placing clips. Stroke subtypes in this animal model are similar to that in SHR-SP: about $1 / 3$ hemorrhage and $2 / 3$ infarction.

\section{REFERENCES}

Cai, G. J., Miao, C.Y., Xie, H. H., Lu, L.H., and Su, D. F. (2005). Arterial baroreflex dysfunction promotes atherosclerosis in rats. Atherosclerosis 183, 41-47.

Daneshtalab, N., and Smeda, J. S. (2010). Alterations in the modulation of cerebrovascular tone and blood flow by nitric oxide synthases in SHRsp with stroke. Cardiovasc. Res. 86, 160-168.

Fang, X. H., Zhang, X. H., Yang, Q. D., Dai, X. Y., Su, F. Z., Rao, M. L., Wu, S. P., Du, X. L., Wang, W. Z., and Li, S. C. (2006). Subtype hypertension and risk of stroke in middle-aged and older Chinese: a 10-year follow-up study. Stroke 37, 38-43.

Franco, V., Oparil, S., and Carretero, O. A. (2004a). Hypertensive therapy: Part I. Circulation 109, 2953-2958.

Franco, V., Oparil, S., and Carretero, O. A. (2004b). Hypertensive therapy: Part II. Circulation 109, 3081-3088.

Fu, Y. J., Shu, H., Miao, C. Y., Wang, M. W., and Su, D. F. (2004). Restoration of baroreflex function by ketanserin is not blood pressure dependent in conscious freely moving rats. J. Hypertens. $22,1165-1172$

Fu, Y. J., Wang, W. Z., Cai, G. J., Wang, M. W., and Su, D. F. (2006). Action site of ketanserin enhancing baroreflex function is within the rostral ventrolateral medulla in anesthetized rats. Auton. Neurosci. 124, 31-37.

Hamaguchi, R., Takemori, K., Inoue, T., Masuno, K., and Ito, H. (2008). Short-term treatment of stroke-prone spontaneously hypertensive rats with an AT1 receptor blocker protects against hypertensive end-organ damage by prolonged inhibition of the renin-angiotensin system. Clin. Exp. Pharmacol. Physiol. 35, 1151-1155.
Henning, E. C., Warach, S., and Spatz, M. (2010). Hypertension-induced vascular remodeling contributes to reduced cerebral perfusion and the development of spontaneous stroke in aged SHRSP rats. J. Cereb. Blood Flow Metab. 30, 827-836.

La Rovere, M. T., Bigger, J. T., Marcus, F. I., Mortara, A., and Schwartz, P. J., for the ATRAMI Investigators. (1998). Baroreflex sensitivity and heart rate variability in prediction of total cardiac mortality after myocardial infarction. Lancet 351, 478-484.

La Rovere, M. T., Specchia, G., Mortara,A., and Schwartz, P. J. (1988). Baroreflex sensitivity, clinical correlates, and cardiovascular mortality among patients with a first myocardial infarction. A prospective study. Circulation 78, 816-824.

Lawes, C. M., Bennett, D. A., Feigin, V. L., and Rodgers, A. (2004). Blood pressure and stroke. an overview of published reviews. Stroke 6, 124-129.

Liu, A. J., Ling, G., Wu, J., Shen, F. M., Wang, D. S., Lin, L. L., Liu, J. G., Su, D. F. (2008). Arterial baroreflex function is an important determinant on the acute cerebral ischemia in rats with middle cerebral artery occlusion. Life Sci 83, 388-393.

Liu, A. J., Ma, X. J., Shen, F. M., Liu, J. G., Chen, H., and Su, D. F. (2007). Arterial baroreflex: a novel target for preventing stroke in rat hypertension. Stroke 38, 1916-1923.

Luepker, R. V., Arnett, D. K., Jacobs, D. R. Jr, Duval, S. J., Folsom, A. R., Armstrong, C., and Blackburn, H. (2006). Trends in blood pressure, hypertension control, and stroke mortality: the Minnesota Heart Survey. Am. J. Med. 119, 42-49.

The results showed that the combination of amlodipine and ketanserin could significantly prolonged survival time in both SHR-SP and 2K2C hypertensive rats. The effects of this combination were significantly greater than those of mono-therapies, even in the smallest dose group (1/2 dose of each drug used in mono-therapy). These results strongly confirmed our hypothesis.

In conclusion, combination of blood pressure reduction and baroreflex restoration possessed a superior preventive effect on stroke. It may be a new strategy for the prevention of stroke in hypertension.

\section{ACKNOWLEDGMENTS}

This study was supported by grants from the National Natural Science Foundation of China (30901809 and 30730106), the National Hi-Technology Research \& Development Program (Project 863, 2006AA02Z4C1), National Basic Research Program of China (973 Program, 2009CB521901).

Papadopoulos, D. P., and Papademetriou, V. (2008). Aggressive blood pressure control and stroke prevention: role of calcium channel blockers. J. Hypertens. 26, 844-852.

Robinson, T. G., Dawson, S. L., Eames, P. J., Panerai, R. B., and Potter, J. F. (2003). Cardiac baroreceptor sensitivity predicts long-term outcome after acute ischemic stroke. Stroke 34, 705-712.

Shen, F. M., Wang, J., Ni, C. R., Yu, J. G., and Su, D. F. (2007). Ketanserininduced baroreflex enhancement in spontaneously hypertensive rats depends on central 5-HT2A receptors. Clin. Exp. Pharmacol. Physiol. 34, 702-707.

Su, D. F., Cerutti, C., Barres, C., Vincent, M., and Sassard, J. (1986). Blood pressure and baroreflex sensitivity in conscious hypertensive rats of Lyon strain. Am. J. Physiol. 251, H1111-H1117.

Wang, T., Takabatake, T., Kobayashi,Y., and Nabika, T. (2008). Sympathetic regulation of renal function in stroke-prone spontaneously hypertensive rats congenic for chromosome 1 blood pressure quantitative trait loci. Clin. Exp. Pharmacol. Physiol. 35, 1365-1370.

Wolf-Maier, K., Cooper, R. S., Banegas, J. R., Giampaoli, S., Hense, H.W., Joffres, M., Kastarinen, M., Poulterm, N., Primatesta, P., Rodriguez-Artalejom, F. Stegmayr, B., Thamm, M., Tuomilehto, J., Vanuzzo, D., and Vescio, F. (2003). Hypertension prevalence and blood pressure levels in 6 European countries, Canada, and the United States. JAMA 289, 2363-2369.

Xie, H. H., Miao, C. Y., Jiang, Y. Y., and Su, D. F. (2005). Synergism of atenolol and nitrendipine on hemodynamic amelioration and organ protection in hypertensive rats. J. Hypertens. 23, 193-201.
Zeng, J.S., Zhang, Y. Q., Mo, J. W., Su, Z. P., and Huang, R. X. (1998). Two-kidney, two clip renovascular hypertensive rats can be used as stroke-prone rats. Stroke 29, 1708-1714.

Zhang, W., Liu, A. J., Yi-Ming, W., Liu, J. G., Shen, F. M., and Su, D. F. (2008) Pressor and non-pressor effects of sodium loading on stroke in strokeprone spontaneously hypertensive rats. Clin. Exp. Pharmacol. Physiol. 35, 83-88.

Conflict of Interest Statement: The authors declare that the research was conducted in the absence of any commercial or financial relationships that could be construed as a potential conflict of interest.

Received: 11 April 2010; paper pending published: 29 April 2010; accepted: 06 May 2010; published online: 29 July 2010.

Citation: Song $S-W$, Liu A-J, Bai C, Su $B-L, M a X-J$, Shen F-M, Duan J-L and Su D-F (2010) Blood pressure reduction combining baroreflex restoration for stroke prevention in hypertension in rats. Front. Pharmacol. 1:6. doi: 10.3389/ fphar.2010.00006

This article was submitted to Frontiers in Cardiovascular and Smooth Muscle Pharmacology, a specialty of Frontiers in Pharmacology.

Copyright (C) 2010 Song, Liu, Bai, Su, Ma, Shen, Duan and Su. This is an open-access article subject to an exclusive license agreement between the authors and the Frontiers Research Foundation, which permits unrestricted use, distribution, and reproduction in any medium, provided the original authors and source are credited. 